\title{
A low complexity partial transmit sequence approach based on hybrid segmentation scheme
}

\author{
Ali Hussein Fadel ${ }^{1}$, Hasanain H. Razzaq ${ }^{2}$, Salama A. Mostafa ${ }^{3}$ \\ ${ }^{1}$ Department of Computer Science, University of Diyala, Iraq \\ ${ }^{2}$ College of Medicine, Jabir Ibn Hayyan University, Iraq \\ ${ }^{3}$ Faculty of Computer Science and Information Technology, Universiti Tun Hussein Onn Malaysia, Malaysia
}

\begin{tabular}{l} 
Article Info \\
\hline Article history: \\
Received Feb 15, 2020 \\
Revised Apr 28, 2020 \\
Accepted May 31, 2020 \\
\hline
\end{tabular}

\section{Keywords:}

Computational complexity

Orthogonal frequency

division multiplexing

Partial transmit sequences

Peak-to-average power ratio

\begin{abstract}
The partial transmit sequences (PTS) is regarded as a promising scheme for inhibiting the high peak-to-average power ratio (PAPR) problem in the orthogonal frequency division multiplexing (OFDM) systems. The PTS scheme relies on partitioning the data sequence into subsets and weighting these subsets by a group of the phase rotation factors. Although the PTS can efficiently reduce the high PAPR value, a great computational complexity (CC) level restricts the utilization of the PTS scheme in practical applications. In PTS, there are three common types of segmentation schemes; interleaving (IL-PTS), pseudo-random (PR-PTS), and adjacent (Ad-PTS) schemes. This paper presents a new algorithm named hybrid pseudo-random and interleaving cosine wave shape (H-PRC-PTS) by combining the PR-PTS scheme and the symmetrical interleaving cosine wave shape (S-IL-C-PTS) scheme which was proposed in our previous work. The results indicate that the suggested algorithms can diminish the PAPR value like the PR-PTS scheme, whereas the CC level is reduced significantly.
\end{abstract}

This is an open access article under the CC BY-SA license.

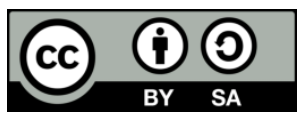

\section{Corresponding Author:}

Salama A. Mostafa,

Faculty of Computer Science and Information Technology,

Universiti Tun Hussin Onn Malaysia,

86400, Parit Raja, Batu Pahat, Johor, Malaysia.

Email: salama@uthm.edu.my

\section{INTRODUCTION}

Recently, orthogonal frequency division multiplexing (OFDM) has imposed its domination in wireless communication frameworks because of the demand for high data rates in many applications of the wireless systems. The OFDM framework is introduced to meet the requirements of the markets, which could not be dealt with the prior wireless systems. OFDM has been adopted by several wireless systems due to its advantages, such as immunity against multipath fading scenario [1], the easy implementation based on the inverse fast Fourier transform (IFFT) algorithm [2], high data rate capacity [3], and efficiency of bandwidth utilization [4]. Therefore, OFDM utilized by many wireless systems like IEEE.802.11 [5], IEEE.802.15 [6], and IEEE.802.16 [7]. Furthermore, OFDM is an essential part of the broadcasting networks, for instance, digital-video-broadcast (DVB) [8], and digital-TV-broadcast (DTVB) [9]. Moreover, OFDM is a multiplexing technique of long-term evolution (LTE), which is the standard of the fourth generation (4G) wireless system [10]. Recently, OFDM based filtering is introduced as the new waveform design candidate in 5th generation (5G) technology, for example, filtered-OFDM (F-OFDM) [11] and universal-filtered multi-carrier (UFMC) systems [12].

On the transmitter side, the main snag encountered the OFDM system is the high PAPR value [13]. When the wireless signal is transmitted with the high PAPR value, some devices of the system, for example, 
the high-power amplifiers (HPA) should be run with a large input back-off to avoid the distortion of the signal. Hence, the change of the HPA characteristics leads to degrading the efficiency and increasing the cost of the system [14]. Therefore, several methods introduced to mitigate the high PAPR challenge, for instance, partial transmitted sequence (PTS) [15], clipping methods [16], tone reservation (TR) [17], selective level mapping (SLM) [18], and companding technique [19]. The PTS technique is considered as one of the effective techniques to reduce the high PAPR value because of its high PAPR reduction capability without influencing to the Bit Error Rate (BER) performance. The PTS is based on partitioning the input symbols of the OFDM system into several subsets and weighting these subsets by a group of phase factors before combining them again. The prominent feature of the PTS algorithm is to reduce the PAPR value more than $3 \mathrm{~dB}$ [20]. Meanwhile, the high computational complexity (CC) level is a significant challenge that faces the PTS technique in real applications [3]. This computational burden is imposed on the system because of the computing of IFFT for the partitioned data and a comprehensive search to identify the best possible phase factor [21].

In literature, numerous works have been published to improve the PAPR lessening effectiveness and restrain the $\mathrm{CC}$ of the system in the PTS technique, such Varahram's algorithm in 2011 [22] that introduced a new set of phase factors to reduce the $\mathrm{CC}$ level with slightly degrading in the PAPR performance. Also, Kim [23] proposed a low CC method by applying a cyclic shifted sequences technique instead of using phase rotation factors to produce an optimum OFDM signal. On the other hand, several algorithms have been introduced for combining the ordinary partitioning schemes to enhance the efficiency of PAPR reduction or reduce the CC level. Most of the combination methods enhance the PAPR reduction performance, which increases the CC level and vice versa [24]. In 1999, Kang [11] proposed a new subblock partitioning scheme named concatenation of interleaving (IL-PTS) and pseudo-random (PR-PTS) schemes. Kang's method could decrease the CC extensively; however, the PAPR lessening performance was less than the PR-PTS method. Moreover, some of the input data sequences were lost, because Kang's method depended on the duplication and concatenation a certain part of the subcarriers within the subblocks. Hence, the BER of the system is degraded. Maio [25] suggested a new method that could inhibit the CC level of the system by combining PR-PTS and IL-PTS with a Hadamard matrix. Despite Maio's method could able reduce the $\mathrm{CC}$ of the system, this approach led to degrading in the PAPR decreasing efficiency.

Also, Hong [13] proposed a method that could reduce the computation complexity significantly by applying PR-PTS on half of the subblocks and IL-PTS for the rest of the subblocks. The problem that accompanied Hong's algorithm is that the PAPR value is larger than that of the PR-PTS, and the algorithm needs four or more of the subblocks for success. In [14], Ibraheem suggested a new partitioning scheme by merging adjacent scheme Ad-PTS and IL-PTS. The hybrid scheme reduced the value of PAPR lower than that of both the IL-PTS and Ad-PTS, while Ibraheem's scheme is restricted using the Ad-PTS scheme. Also, Jawhar in [7] presented a new partitioning scheme to enhance the PAPR diminishing performance better than the Ad-PTS and IL-PTS schemes alternately.

In addition, Jawhar in [8] introduced two schemes to promote the PAPR reduction performance of both the Ad-PTS and IL-PTS schemes, the first scheme depended on dividing the subblocks of the Ad-PTS matrix into several groups and then shifting its subcarriers, whereas the second scheme depended on dividing the subblocks of the IL-PTS matrix into two identical matrices and then exchanging the even and the odd parts with each other. Although the shifting and exchanging methods improved the PAPR reduction performance, the mathematical calculations are still the same as those of the Ad-PTS scheme. Lastly, Jawhar in [15] enhanced new segmentation schemes to improve the performance of IL-PTS by reducing the PAPR value. Jawhar's approaches were based on reshaping the IL-PTS matrix to produce new matrices, which have subcarriers distribution identical to that of the IL-PTS matrix, but with farther PAPR lessening performance. It evident from the aforementioned methods, there is no perfect scheme can accomplish PAPR reduction performance matches the PR-PTS scheme with low CC level. Therefore, this paper introduces a new combination method that can accomplish PAPR lessening performance that matches that of the PR-PTS scheme with a considerable reducing in the CC level.

\section{METHODS}

The proposed method in this paper is an integration of PR-PTS and S-IL-C-PTS schemes in parallel; therefore, the merged method is called the hybrid pseudo-random-cosine wave shape scheme (H-PRC-PTS). The H-PRC-PTS algorithm employs the distinctive features of each segmentation scheme to decrease the CC level significantly without retreating the PAPR performance lower than PR-PTS.

\subsection{OFDM system}

In the OFDM system, the input data symbols are firstly mapped by baseband modulation schemes, for instance, quadrature amplitude modulation (QAM). Then, the modulated symbols $\mathrm{Yk}\{\mathrm{k}=0,1,2, \ldots, \mathrm{N}-1\}$ Bulletin of Electr Eng \& Inf, Vol. 9, No. 6, December 2020 : 2371 - 2379 
are transformed into the time-domain (TD) by using IFFT operations to produce the discrete baseband OFDM signal $y(n)$, which can be depicted as [14].

$$
y(n)=\frac{1}{\sqrt{N}} \sum_{k=0}^{N-1} Y_{k} e^{j 2 \pi k \frac{n}{N}} \quad, \quad n=0,1,2, \ldots, N-1
$$

where $\mathrm{y}$ and $\mathrm{N}$ symbolize the number of subcarriers. The discrete baseband OFDM signal represents the superposition of k-samples of the input data symbol with $\mathrm{N}$-subcarriers orthogonally.

In the TD, the output OFDM signal is produced by summing the $\mathrm{N}$ - modulated samples together. Thus, the instantaneous power of some samples increases considerably larger than the average power of the signal; this is done when the phases of these samples are similar. This fluctuation of the signal is that so-called PAPR, and it is expressed by dividing the maximum instantaneous power into the mean power of the signal. The PAPR is usually expressed in decibels $(\mathrm{dB})$ [13].

$$
\operatorname{PAPR}=\frac{\max |y(n)|^{2}}{E\left\{|y(n)|^{2}\right\}}
$$

where E represents the mean value. If the OFDM signal is transmitted with a high PAPR value, some components of the system like HPA will be driven into the non-linear region. Hence, the superposition of the signals will be changed, and this leads to degradation in the spectral efficiency and retraction in the BER performance [15].

In the case of dealing with PAPR calculations, the oversampling factor (L) should be employed to ensure the PAPR calculations are more accurate, because some peaks of the samples may disappear. Thus, the oversampling operation gives the discrete-time signal similar characteristics to those of the continuous-time signal. The oversampling operation can be fulfilled by adding (L-1) N zeros among the samples [17]. Moreover, the complementary cumulative distribution function (CCDF) usually used to evaluate the probability of PAPR values that exceeds a specific threshold value (PAPR0).

$$
\operatorname{Pr}\left(\operatorname{PAPR}>P A P R_{0}\right)=1-\left(1-\exp \left(-P A P R_{0}\right)\right)^{N L}
$$

\subsection{Conventional PTS (C-PTS)}

PTS is addressed as a probabilistic method that can mitigate the PAPR value more than $3 \mathrm{~dB}$ when using the PR-PTS scheme without degrading in the BER performance [3]. The principal idea of C-PTS is the segmentation of the input data block into V subblocks.

$$
Y=\sum_{v=1}^{V} Y_{v}
$$

where the number of subblocks is represented by V. Afterwards, the N-IFFT operation is performed on the subblocks to modulate the input data with the subcarriers orthogonally; with the consideration of inserting zeros instead of the null subcarriers to complete the length of the N-IFFT. Next, the subblocks in the TD are multiplied by a set of phase factor vectors (bv) component-wise, to produce a group of candidate sequences. Equation (5) expresses the OFDM signal.

$$
y=\operatorname{IFFT}\left\{\sum_{v=1}^{V} b_{v} Y_{v}\right\}
$$

The linear property of IFFT is exploited to convert bv into the TD because the amplitude of the phase rotation factors is fixed to one; thus, the output OFDM signal updated to,

$$
y=\sum_{v=1}^{V} b_{v} \operatorname{IFFT}\left\{Y_{v}\right\}
$$

where $\mathrm{v}=\{1,2, \ldots, \mathrm{V}\}$, and bv represents the phase rotation factors. Finally, the PAPR value is computed of every candidate sequence, whereas the candidate that has the lowest PAPR value is elected for transmission. The output OFDM signal that utilizes the PTS technique can be expressed as [18]: 


$$
\text { OFDM signal }=\sum_{v=1}^{V} b_{v} y_{v}
$$

It can be concluded from the following Figure 1 that PTS relies on the partitioning schemes, as will be explained in the next section, and the phase rotation factors, which are usually limited to bv $\in\{ \pm 1\}$ or $\{ \pm 1, \pm \mathrm{j}\}$ to decrease the complex multiplications [19]. On the other hand, the phase rotation factors can be obtained as:

$$
b=\left\{b_{v}=e^{j 2 \pi / W} \mid v=0,1, \ldots \ldots, W-1\right\}
$$

where the number of the various phase rotation factor is symbolized by $\mathrm{W}$. The optimum phase weighting factors can be found by using the following optimization expression:

$$
\left\{b_{1}, b_{2}, \ldots \ldots, b_{v}\right\}=\underset{1 \leq w \leq W}{\arg \min }\left(\max _{0 \leq n \leq N L-1}\left|\sum_{v=1}^{V} b_{v} y_{v}\right|\right)
$$

In C-PTS, the system should perform a comprehensive search to determine the optimum phase factor, so this operation imposes a high $\mathrm{CC}$ level, and it requires high processing time. Moreover, the transmitter must send $(\log 2 \mathrm{WV}-1)$ bits to the receiver as side information to extract the transmitted data at the receiver side [20, 26]. It is noted that the CC level of PR-PTS and Ad-PTS differs to IL-PTS because all the IFFT stages are required to pass to process the data subblocks inside the IFFT unit, whereas the IL-PTS scheme requires fewer stages to convert the subblocks from the frequency-domain (FD) into the TD. Consequently, the number of additions and multiplications of IL-PTS is less than that PR-PTS and Ad-PTS, but at the expense of PAPR reduction efficiency degradation. These observations motivated us to develop our approach, which will be shown in the next section. Figure 1 shows the C-PTS block diagram.

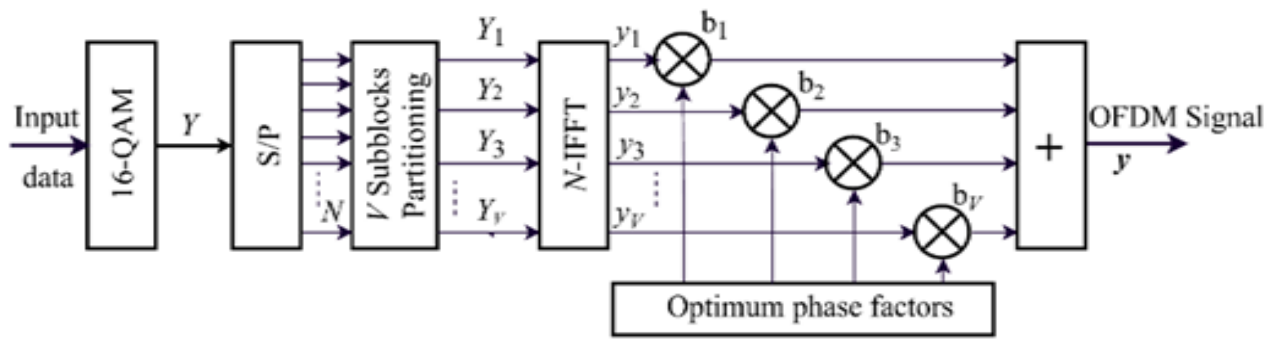

Figure 1. C-PTS block diagram [3]

The superior PAPR reduction capacity is observed by the PR-PTS scheme comparing with the other segmentation schemes, but this feature is accompanied by a high CC level. But, IL-PTS has the lowest CC among the conventional schemes at the expense of the PAPR performance degeneration. Therefore, the combination of the PR-PTS with the IL-PTS segmentation schemes in parallel may accomplish a low number of mathematical operations, but the PAPR lessening performance will be degraded compared with the PR-PTS algorithm $[19,27]$. In this paper, the objective is to diminish the CC level without retreating of the PAPR performance. To achieve this target, two segmentation schemes are employed, the PR-PTS scheme and symmetrical interleaving cosine wave shape scheme (S-IL-C-PTS), which was proposed in the previous study $[18,25]$.

\subsection{S-IL-C-PTS scheme}

The S-IL-C-PTS scheme was presented to evaluate the PAPR lessening gain of the IL-PTS scheme with slightly additional computation complexity [21, 22, 26]. The S-IL-C-PTS depends on reshaping the IL-PTS matrix symmetrically to produce an original matrix, where the subcarriers are distributed in the shape of a cosine wave. The S-IL-C-PTS scheme starts when the columns of the IL-PTS matrix are partitioned into $\mathrm{SG}$ groups, and $\mathrm{V}$ columns are included in every group, where the subscript $\mathrm{G}=\{1,2,3, \ldots$, $\mathrm{N} / \mathrm{V}\}$. After that, only the even $\mathrm{SG}\{\mathrm{G}=2,4,6, \ldots, \mathrm{N} / \mathrm{V}\}$ groups are selected for processing. The first row of every even group is replaced with the last row while the second row is changed by the row one before the last, and so forth. As a result, another matrix is generated from the IL-PTS matrix is, where 
the symmetrical distribution of the subcarriers is formed as cosine waves [16, 28]. To understand the S-IL-CPTS scheme, the example below illustrates the S-IL-C-PTS scheme procedure when $\mathrm{N}=16$, and $\mathrm{V}=4$. The IL matrix is established first as follow:

$$
\text { IL-matrix }=\left[\begin{array}{cccccccccccccccc}
Y_{1} & 0 & 0 & 0 & Y_{5} & 0 & 0 & 0 & Y_{9} & 0 & 0 & 0 & Y_{13} & 0 & 0 & 0 \\
0 & Y_{2} & 0 & 0 & 0 & Y_{6} & 0 & 0 & 0 & Y_{10} & 0 & 0 & 0 & Y_{14} & 0 & 0 \\
0 & 0 & Y_{3} & 0 & 0 & 0 & Y_{7} & 0 & 0 & 0 & Y_{11} & 0 & 0 & 0 & Y_{15} & 0 \\
0 & 0 & 0 & Y_{4} & 0 & 0 & 0 & Y_{8} & 0 & 0 & 0 & Y_{12} & 0 & 0 & 0 & Y_{16}
\end{array}\right]
$$

After that, the IL matrix is partitioned into SG groups, denoted by $\{\mathrm{S} 1, \mathrm{~S} 2, \mathrm{~S} 3, \mathrm{~S} 4\}$ groups, as follows:

$$
\text { IL-matrix }=\left[\begin{array}{cccc|cccc|cccc|cccc}
S_{1} & 0 & 0 & 0 & Y_{5} & 0 & 0 & 0 & Y_{9} & 0 & 0 & 0 & Y_{13} & 0 & 0 & 0 \\
0 & Y_{2} & 0 & 0 & 0 & Y_{6} & 0 & 0 & 0 & Y_{10} & 0 & 0 & 0 & Y_{14} & 0 & 0 \\
0 & 0 & Y_{3} & 0 & 0 & 0 & Y_{7} & 0 & 0 & 0 & Y_{11} & 0 & 0 & 0 & Y_{15} & 0 \\
0 & 0 & 0 & Y_{4} & 0 & 0 & 0 & Y_{8} & 0 & 0 & 0 & Y_{12} & 0 & 0 & 0 & Y_{16}
\end{array}\right]
$$

Next, only the even groups $\{S 1, S 2\}$ are chosen for processing, where the rows of each even group are exchanged with each other; thus, the S-IL-C matrix can be expressed as:

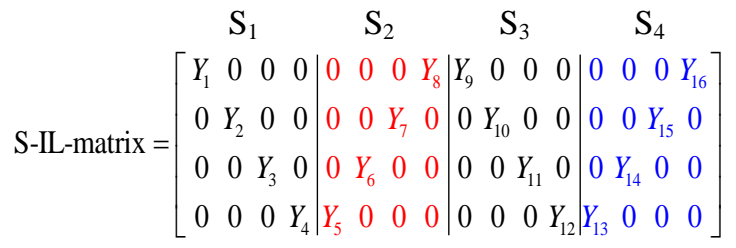

Finally, the S-IL-C matrix can be written as:

$$
\text { S-IL-C-matrix }=\left[\begin{array}{cccccccccccccccc}
Y_{1} & 0 & 0 & 0 & 0 & 0 & 0 & Y_{8} & Y_{9} & 0 & 0 & 0 & 0 & 0 & 0 & Y_{16} \\
0 & Y_{2} & 0 & 0 & 0 & 0 & Y_{7} & 0 & 0 & Y_{10} & 0 & 0 & 0 & 0 & Y_{15} & 0 \\
0 & 0 & Y_{3} & 0 & 0 & Y_{6} & 0 & 0 & 0 & 0 & Y_{11} & 0 & 0 & Y_{14} & 0 & 0 \\
0 & 0 & 0 & Y_{4} & Y_{5} & 0 & 0 & 0 & 0 & 0 & 0 & Y_{12} & Y_{13} & 0 & 0 & 0
\end{array}\right]
$$

The realized PAPR reduction of S-IL-C-PTS surpasses that of the IL-PTS scheme due to the observed autocorrelation among the subcarriers of IL-PTS is higher than that of S-IL-C-PTS [16]. In contrast, the CC of S-IL-C-PTS is calculated based on the divide-and-conquer approach combined with the Cooley-Tukey IFFT algorithm. In such a way, according to the column-wise mapping, every subblock of the S-IL-C-PTS scheme is arranged, in which in the S-IL-C-PTS scheme, the subblocks V has the same number of rows. While, dividing each subblock into subsets calculates the number of columns, in which the entire number of subsets $\mathrm{R}=\mathrm{N} / \mathrm{V}$, as demonstrated in Table 1 . However, the generated signal of every subblock is restored a row-wise mapping based on the divide-and-conquer approach.

Table 1. The subblock arrangement of the S-IL-C-PTS scheme [11]

\begin{tabular}{lllll}
\hline$v \backslash \mathrm{r}$ & 1 & 2 & $\ldots$ & $R$ \\
\hline 1 & $Y_{1}^{v}$ & $Y_{V+1}^{v}$ & $\cdots$ & $Y_{V(R-1)+1}^{v}$ \\
2 & $Y_{2}^{v}$ & $Y_{V+2}^{v}$ & $\ldots$ & $Y_{V(R-1)+2}^{v}$ \\
$\ldots$ & $\ldots$ & $\ldots$ & $\ldots$ & $\ldots$ \\
$V$ & $Y_{V}^{v}$ & $Y_{2 V}^{v}$ & $\cdots$ & $Y_{V R}^{v}$ \\
\hline
\end{tabular}

The number of rows in Table 1 is equalled to the subblocks number, and $\mathrm{R}$ represents the number of subsets in a single subblock. In the TD, the subblock data sequence is obtained after employing the Cooley-Tukey scheme on each row and column in Table 1 . Therefore, the TD signal can be represented as [11, 23]: 


$$
y_{(q, p)}=\frac{1}{\sqrt{N}} \sum_{v=0}^{V-1}\left\{W_{N}^{v q}\left[\sum_{r=0}^{R-1} Y_{v, r} W_{R}^{r q}\right]\right\} W_{V}^{v p}
$$

where $\left(W_{N}=\mathrm{e}^{2 j \pi / N}\right)$ is the twiddle factor, while the elements of the $\mathrm{p}^{\text {th }}$ row and $\mathrm{q}^{\text {th }}$ column of the row-wise mapping of IFFT signal $\mathrm{y}_{(\mathrm{q}, \mathrm{p})}$, respectively. Moreover, the scope of the rows and columns are $1 \leq p \leq V$ and $1 \leq q \leq R$ respectively. According to the approach of the S-IL-C-PTS scheme, only two rows that have the active subcarriers of subsets will be applied R-point IFFT. Therefore, the expression in (1) can be simplified as [11]:

$$
y_{(p, q)}=\frac{1}{\sqrt{N}} \sum_{v=0}^{1}\left\{W_{N}^{v q}\left[\sum_{r=0}^{R-1} Y_{(v, r)} W_{R}^{r q}\right]\right\} W_{V}^{v p}
$$

Consequently, the S-IL-C-PTS scheme can realize better PAPR reduction gain than that of IL-PTS with slightly increasing of mathematical operations, which motivated us to exploit this prominent feature in the proposed method in this paper.

\subsection{PR-PTS scheme}

As mentioned in Section 4, the superior algorithm among the conventional partitioning schemes regarding the PAPR lessening performance is the PR-PTS scheme, because the random distribution of the subcarriers within the subblocks leads to decreasing the correlation between the subcarriers [15, 24]. Although the PR-PTS algorithm can be restrained the PAPR value significantly, the large number of mathematical operations is considered the main shortcoming of this technique, because the PR-PTS scheme utilizes the entire stages of the IFFT unit during transforming the subblocks. Accordingly, the PR-PTS method is the preferred algorithm when the system needs a high level of extent PAPR reduction performance; therefore, this merit can be employed for the proposed method in this paper.

\subsection{H-PRC-PTS scheme}

As mentioned in the first part of this section, the H-PRC-PTS algorithm merges the PR-PTS scheme and the S-IL-C-PTS scheme to produce a hybrid scheme that can reduce the number of mathematical operations significantly, and also it accomplishes the PAPR lessening performance equal to the PR-PTS scheme. The H-PRC-PTS method is suggested to make use of the high PAPR reduction capability of PR-PTS and the low CC level feature of S-IL-C-PTS. The block diagram of the H-PRC-PTS method is demonstrated in Figure 1, where the first step begins by dividing the input data symbol $\mathrm{Y}$ into two parts $\mathrm{Y}_{\mathrm{A}}$ and $\mathrm{Y}_{\mathrm{B}}$.

$$
Y=\left[Y_{1}, Y_{2}, \ldots, Y_{N}\right]
$$

therefore, the first step can be expressed as:

$$
Y=\left[Y_{A}, Y_{B}\right]
$$

where YA represents the first part of the input data symbol and contains $0.25 \mathrm{~N}$ or $0.5 \mathrm{~N}$ or $0.75 \mathrm{~N}$ of the subcarriers. For the sake of simplicity in the illustration figure supposes that the input data symbol $\mathrm{Y}$ is split into two equal parts, $0.5 \mathrm{~N}$ each; therefore, YA can be defined as:

$$
Y_{A}=\left[Y_{1}, Y_{2}, \ldots, Y_{N / 2}\right]
$$

The second part of the input symbol YB can be written as

$$
Y_{B}=\left[Y_{(N / 2)+1}, Y_{(N / 2)+2}, \ldots, Y_{N}\right]
$$

\section{RESULTS AND DISCUSSION}

In this part, the proposed H-PRC-PTS method is simulated and analyzed in one scenario, depending on the number of subcarriers which are determined in the first part $\left(\mathrm{Y}_{\mathrm{A}}\right)$ and the second part $\left(\mathrm{Y}_{\mathrm{B}}\right)$. In this simulation, the first part is applied to PR-PTS and the second part is applied to S-IL-C-PTS, where the number of subcarriers for $\mathrm{Y}_{\mathrm{A}}$ is $N^{\prime}$ and $\mathrm{YB}$ is $N^{\prime \prime}$. Subsequently, where the number of subcarriers $\left(N^{\prime}, N^{\prime \prime}\right)$ will be set to $(\mathrm{N} / 2, \mathrm{~N} / 2),(\mathrm{N} / 4,3 \mathrm{~N} / 4)$, and $(3 \mathrm{~N} / 4, \mathrm{~N} / 4)$, respectively. Moreover, the proposed 
method in the scenario is compared with PR-PTS. Besides, the parameters that are used to conduct the simulation are, the number of subcarriers $\mathrm{N}=256, \mathrm{~L}=4$, and 16-QAM family to modulate 1000 random symbols which are evaluated by the CCDF function [28]. Moreover, different numbers of the subblocks V and phase rotation factors $\mathrm{W}$ are utilized for this simulation, and they are set as (W, V) to $(2,4),(2,8),(4,2)$, and $(4,4)$, respectively. On the other hand, the CC of PR-PTS, H-PRC-PTS, and the CC reduction ratio (CCRR) are tested and evaluated in two scenarios. The CCRR between the proposed method and the PR-PTS method can be written as [21].

$$
\text { CCRR }=1-\frac{\text { Complexity of H-PRC-PTS }}{\text { Complexity of PR-PTS }} \times 100 \%
$$

The simulation is conducted when the number of subcarriers for YA and $\mathrm{YB}\left(N^{\prime}, N^{\prime \prime}\right)$ is set to $(\mathrm{N} / 2, \mathrm{~N} / 2)$. In this scenario, PR-PTS is compared with the proposed method which is symbolized by H-PRC1-PTS. Furthermore, the number of the subcarriers $\mathrm{N}=256$ and the $(\mathrm{W}, \mathrm{V})$ parameters are set to $(2,4)$, $(2,8),(4,2)$, and $(4,4)$, respectively. Figure 2(a) illustrates the comparison of H-PRC1-PTS and PR-PTS when $\mathrm{W}=2$, and $\mathrm{V}=4$. The PAPR value of $\mathrm{H}-\mathrm{PRC} 1-\mathrm{PTS}$ is equivalent to that of PR-PTS at $8.471 \mathrm{~dB}$. Moreover, the PAPR value of PR-PTS is $7.419 \mathrm{~dB}$ which is similar to the PAPR value of H-PRC1-PTS when $\mathrm{W}=2$ and $\mathrm{V}=8$, as displayed in Figure 2(b).

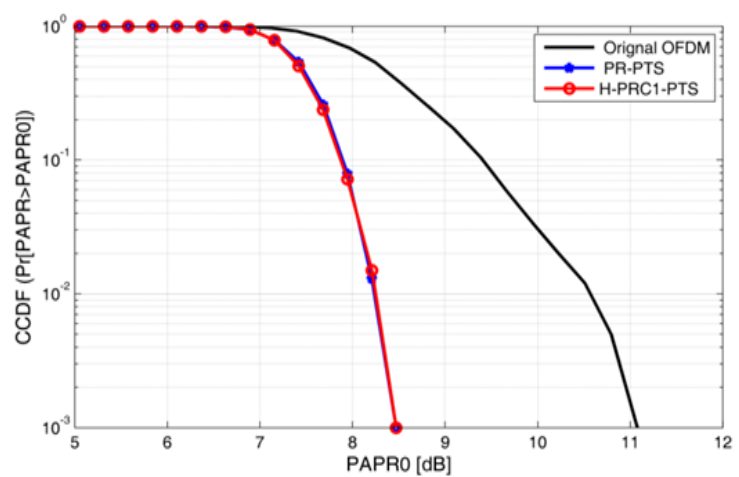

(a)

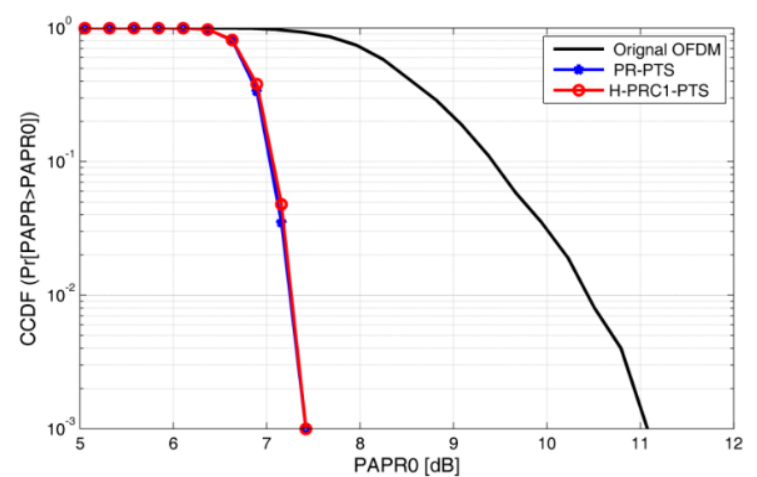

(b)

Figure 2. The PAPR of the H-PRC1-PTS and PR-PTS in the first scenario, (a) W=2, and V=4, (b) $\mathrm{W}=2$, and $\mathrm{V}=8$

Likewise, the simulation result in Figure 3(a) clarifies that PR-PTS and the H-PRC1-PTS can accomplish the same value of PAPR reduction, $9.26 \mathrm{~dB}$, with the consideration that $\mathrm{W}=4$ and $\mathrm{V}=2$. The last simulation of this scenario is conducted when $\mathrm{W}=4$ and $\mathrm{V}=4$, as shown in Figure 3(b), where the PAPR value of both algorithms, PR-PTS and H-PRC1-PTS, is 7.682dB. Consequently, the proposed method in this scenario can accomplish the same PAPR reduction performance of PR-PTS for various numbers of W, and V.

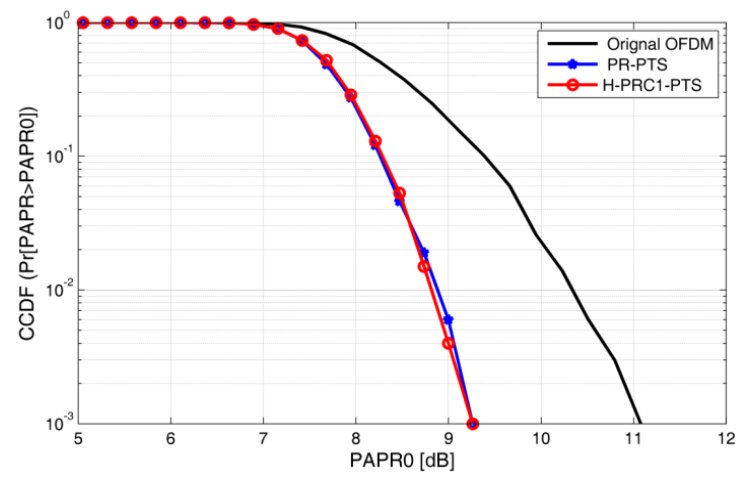

(a)

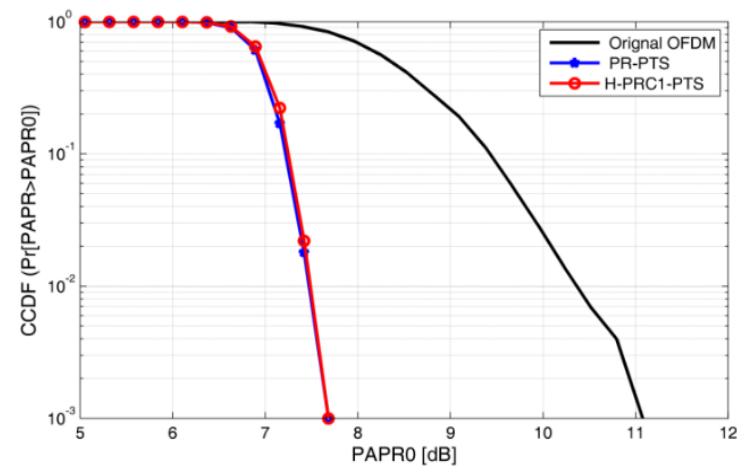

(b)

Figure 3. The PAPR of the H-PRC1-PTS and PR-PTS in the second scenario, (a) W=4, and V=2, (b) $\mathrm{W}=4$, and $\mathrm{V}=4$ 


\section{CONCLUSION}

The paper proposes a new combination method of the C-PTS technique in OFDM systems. The enhanced method, H-PRC-PTS, combines two kinds of segmentation schemes in parallel; the first one is PR-PTS which has the superior PAPR reduction performance with a high CC level, and the second is S-IL-CPTS which has an acceptable PAPR lessening performance with a low CC level. The simulation results are conducted for three scenarios depending on the number of subcarriers in each segmentation scheme. The results show the superiority of the enhanced method over the best well-known segmentation scheme (PR-PTS) regarding PAPR gain and CC. Therefore, the new algorithm could be the best combination method to restrain the high CC level of the C-PTS technique without deterioration in the PAPR gain.

\section{ACKNOWLEDGEMENTS}

This research is supported by the Department of Computer Science, University of Diyala. It is also supported by Research Fund to E15501, Research Management Center, Universiti Tun Hussein Onn Malaysia (UTHM).

\section{REFERENCES}

[1] X. Wu, J. Wang, Z. Mao, and J. Zhang, "Conjugate interleaved partitioning PTS scheme for PAPR reduction of OFDM signals," Circuits, Systems and Signal Processing, vol. 29, no. 3, pp. 499-514, 2010.

[2] Y. Jawhar, L. Audah, M. A. Taher, K. N. Ramli, n. S. M. Dhah, M. Musa, and M. S. Ahmed, "A review of partial transmit sequence for PAPR reduction in the OFDM system," IEEE Access, vol. 7, no. 1, pp. 18021-18041, 2019.

[3] M. A. Taher, J. S. Madeep, M. Ismail, S. A. Samad, and M. T. Islam, "Reducing the power envelope fluctuation of OFDM systems using side information supported amplitude clipping approach," International Journal of Circuit Theory and Applications, vol. 42, no. 4, pp. 425-435, 2014.

[4] H. Liang, H. C. Chu, and C. B. Lin, "Peak-to-average power ratio reduction of orthogonal frequency division multiplexing systems using modified tone reservation techniques,"International Journal of Communication Systems, vol. 29, no. 4, 748-759, 2016.

[5] M. A. Taher, M. J. Singh, M. Ismail, S. A. Samad, M. T. Islam, and H. F. Mahdi, "Post-IFFT-modified selected mapping to reduce the PAPR of an OFDM system," Circuits, Systems, and Signal Processing, vol. 34, no. 2, pp. 535-555, 2015.

[6] S. Jeng, J. M. Chen, and P. H. Chang, "A low complexity companding technique using Padé approximation for PAPR reduction of an OFDM system," International Journal of Communication Systems, vol. 24, no. 11, pp. 1467-1479, 2011.

[7] Y. Al-Jawhar, K. N. Ramli, A. Mustapha, S. A. Mostafa, N. S. M. Shah, and M. A. Taher, "Reducing PAPR with low complexity for 4G and 5G waveform designs," IEEE Access, ; vol. 7, no. 1, pp. 97673-97688, 2019.

[8] Y. Jawhar, K. Ramli, M. Taher, L. Audah, N. S. Shah, M. Ahmed, and A. Hammoodi, "An enhanced partial transmit sequence based on combining hadamard matrix and partitioning schemes in OFDM systems," Inter. J. of Integrated Engineering, vol. 10, no, 3, pp. 1-7, 2018.

[9] P. Varahram and B. Ali, "A low complexity partial transmit sequence for peak to average power ratio reduction in OFDM systems," Radioengineering, vol. 20, no. 3, pp. 677-682, 2011.

[10] K-H. Kim, "On the shift value set of cyclic shifted sequences for PAPR reduction in OFDM systems," IEEE Transactions on Broadcasting, vol. 62, no. 2, pp. 496-500, 2015.

[11] S. G. Kang, J. G. Kim, and E. K. Joo, "A novel subblock partition scheme for partial transmit sequence OFDM," IEEE Transactions on Broadcasting, vol. 45, no. 3, pp. 333-338, 1999.

[12] L. Miao and Z. Sun, "PTS algorithm for PAPR suppression of WOFDM system," International Conference on Mechatronic Sciences, Electric Engineering and Computer (MEC), pp. 1144-1147, 2013.

[13] C. Hong, Q. Qin, and T. Chao, "An PTS optimization algorithm for PAPR reduction of OFDM system," IEEE Int. Conference on Mechatronic Sciences, Electric Engineering and Computer MEC, pp. 3775-3778, 2013.

[14] Z. T. Ibraheem, M. M. Rahman, S. N. Yaakob, M. S. Razalli, F. Salman, and K. K. Ahmed, "PTS method with combined partitioning schemes for improved PAPR reduction in OFDM system,". Indonesian Journal of Electrical Engineering and Computer Science, vol. 12, no. 11, 7845-7853, 2014.

[15] Y. Al-Jawhar, K. N. Ramli, M. A. Taher, N. S. Mohd Shah, L. Audah, and M. S. Ahmed, "A new subblock segmentation scheme in partial transmit sequence for reducing PAPR value of the OFDM systems," Int. J. of Integrated Engineering, vol. 10, no. 8, pp. 91-97, 2019.

[16] Y. Jawhar, N. S. Shah, M. A. Taher, M. Ahmed, K. Ramli, and R. Abdulhasan, "A low PAPR performance with new segmentation schemes of partial transmit sequence for OFDM systems," IJAAS International Journal of Advanced and Applied Sciences, vol. 4, no. 4, pp. 14-21, 2017.

[17] Y. Al-Jawhar, N. S. M. Shah, M. A. Taher, M. S. Ahmed, and K. N. Ramli, "An enhanced partial transmit sequence segmentation schemes to reduce the PAPR in OFDM systems,". International Journal of Advanced Computer Science and Applications (IJACSA), vol. 7, no. 12, pp. 66-75, 2017.

[18] A. M. Elshirkasi, M. U. Siddiqi, and M. H. Habaebi, "Generalized discrete fourier transform based improvement of partial transmit sequence scheme to reduce PAPR in OFDM systems," Journal of Theoretical \& Applied Information Technology, vol. 76, no. 1, pp. 76-81, 2015. 
[19] H. Hung, "Using evolutionary computation technique for trade-off between performance peak-to average power ration reduction and computational complexity in OFDM systems," Computers and Electrical Engineering, vol. 37, no. 1, pp. 57-70, 2011.

[20] H. S. Joo, K. H. Kim, J. S. no, and D. J. Shin, "New PTS schemes for PAPR reduction of OFDM signals without side information," IEEE Transactions on Broadcasting, vol. 63, no. 3, pp. 562-570, 2017.

[21] L. Yang, K-K. Soo, S. Q. Li, and Y. M. Siu, "PAPR reduction using low complexity PTS to construct of OFDM signals without side information," IEEE Transactions on Broadcasting, vol. 57, no. 2, pp. 284-290, 2011.

[22] S. I. Kamarudin, A. Ismail, A. Sali, and Mohd Y. Ahmad, "Magnetic resonance coupling for 5G WPT applications," Bulletin of Electrical Engineering and Informatics, vol. 8, no. 3, pp. 1036-1046, 2019.

[23] M. Njikam, S. Nanna, S. Shahrin, and M. F. I. Othman, "High speed internet development in Africa using 4G-LTE technology-a review," Bulletin of Electrical Engineering and Informatics, vol. 8, no. 2, pp. 577-585, 2019.

[24] S. A. Mostafa, A. Mustapha, P. Shamala, O. I. Obaid, and B. A. Khalaf, "Social networking mobile apps framework for organizing and facilitating charitable and voluntary activities in Malaysia," Bulletin of Electrical Engineering and Informatics, vol. 9, no. 2, pp. 827-833, 2020.

[25] M. H. Hassan, S. A. Mostafa, M. A. Mohammed, D. A. Ibrahim, B. A. Khalaf, and A. S. Al-Khaleefa, "Integrating African Buffalo optimization algorithm in AODV routing protocol for improving the QoS of MANET," Journal of Southwest Jiaotong University, vol. 54, no. 3, 2019.

[26] A. M. Al-Momani, M. A. Mahmoud, and M. S. Ahmad, "Identification of factors influencing customer acceptance and use of IoT services," Advanced Science Letters, vol. 24, no. 10, pp. 7428-7432, 2018.

[27] M. Gurunathan and M. A. Mahmoud, "A review and development methodology of a lightweight security model for IoT-based smart devices," International Journal of Advanced Computer Science and Applications, vol. 2, pp. 125-134, 2020.

[28] F. S. Shawqi, L. Audah, S. A. Mostafa, S. S. Gunasekaran, A. Baz, A. T. Hammoodi, H. Alhakami, M. H. Hassan, M. A. Jubair, and W. Alhakami, "A new SLM-UFMC model for universal filtered multi-carrier to reduce cubic metric and peak to average power ratio in 5G technology," Symmetry, vol. 12, no. 6, p. 909, 2020. 